\section{Commentary: Cut your coat according to your cloth}

\author{
Dhiraj P. Singh, MD, ${ }^{\mathrm{a}}$ and Robert D. B. Jaquiss, $\mathrm{MD}^{\mathrm{b}}$
}

Anomalous aortic origin of a coronary artery (AAOCA) poses an uncertain but increased risk for sudden cardiac arrest (SCA). Balancing this peril with hazards of cardiac surgery remains challenging. ${ }^{1}$ Expert guidelines provide recommendations on indications and timing for AAOCA repair. ${ }^{2}$ Multiple techniques have been described for repair: unroofing of the intramural segment to relocate the ostium, direct reimplantation of the AAOCA, creation of a neoostium, and even coronary artery bypass grafting. ${ }^{3}$ All these techniques aim to provide normal coronary flow at rest and under stress; however, which technique is appropriate for which patient remains unsettled. In this context, the study by Bonilla-Ramirez and colleagues ${ }^{4}$ provides insight into technique choice from a group that has taken the lead in the organized approach to patients with AAOCA. The authors prefer unroofing if the intramural course is distal to the commissural pillar of the aortic valve and there is a suitable exit site of the AAOCA from the aortic wall. If these criteria are not met, or if the unroofing has been judged unsatisfactory by the surgeon, the AAOCA is transected and reimplanted (TAR) into the "correct" sinus.

Using this tailored approach in 61 consecutive patients over 7 years, the authors report essentially identical and excellent outcomes in patients undergoing unroofing $(\sim 75 \%)$ and TAR $(\sim 25 \%)$. There was "crossover" between the groups, with 6 patients undergoing intraoperative conversion from unroofing to TAR, though this was less common later in the study period. There were no deaths. One TAR patient required coronary artery bypass grafting for

From the ${ }^{\mathrm{a}}$ Division of Pediatric Cardiology, Department of Pediatrics and ${ }^{\mathrm{b}}$ Division of Pediatric Cardiothoracic Surgery, Department of Cardiovascular and Thoracic Surgery, University of Texas Southwestern Medical Center and Children's Medical Center, Dallas, Tex.

Disclosures: The authors reported no conflicts of interest.

The Journal policy requires editors and reviewers to disclose conflicts of interest and to decline handling or reviewing manuscripts for which they may have a conflict of interest. The editors and reviewers of this article have no conflicts of interest.

Received for publication Jan 12, 2021; revisions received Jan 12, 2021; accepted for publication Jan 13, 2021; available ahead of print Jan 20, 2021.

Address for reprints: Robert D. B. Jaquiss, MD, Children's Medical Center, 1935 Medical District Dr, Dallas, TX 75235 (E-mail: Robert.Jaquiss@ UTSouthwestern.edu).

J Thorac Cardiovasc Surg 2021;162:1200-1

$0022-5223 / \$ 36.00$

Copyright (c) 2021 by The American Association for Thoracic Surgery

https://doi.org/10.1016/j.jtcvs.2021.01.053

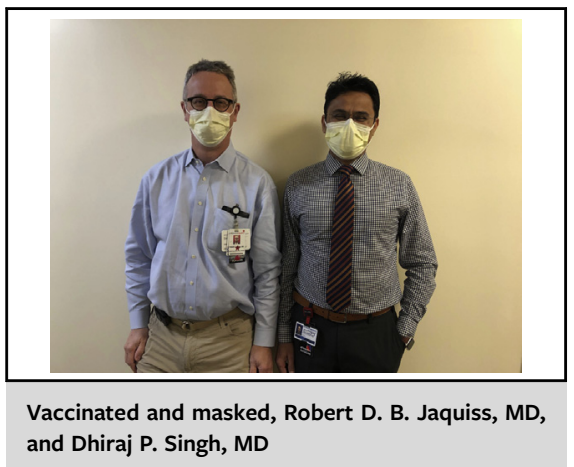

CENTRAL MESSAGE

For patients with anomalous

aortic origin of a coronary artery,

transection and reimplantation

into the "correct" aortic sinus

provides excellent outcomes,

equivalent to the "unroofing"

procedure.

persistent ischemia, and 1 unroofing patient required late reoperation for SCA because of previously unrecognized myocardial bridging. Persistence of symptoms and/or abnormal postoperative stress testing was equivalent and very low in the 2 groups, and in both groups, nearly $95 \%$ of patients received exercise clearance.

When considering the lessons of the present work, several limitations must be noted. As the authors acknowledge, adoption of TAR as a "first choice" approach took some time, even among surgeons experienced with AAOCA. TAR requires longer cross-clamp and bypass times and creates substrate for anastomotic stenosis (1 of 16 in this early experience). Moreover, criteria for electing TAR remain seem to depend heavily on intraoperative judgment rather than on more objective preoperative imaging. "Game time decisions" are necessary in surgery but should be minimized whenever possible. Perhaps most importantly, follow-up is short (median, 4 years), and the value of postoperative stress testing remains uncertain. Shortterm success has been demonstrated, but lifelong success (elimination of elevated SCA risk) has not.

With the obligatory caveats completed, the authors are to be congratulated for presenting a safe and effective approach for AAOCA patients in whom the simpler alternative of unroofing may jeopardize the aortic valve or be ineffective. Their rigorous, protocol-driven approach to AAOCA patients has set an enviable standard. It must be emphasized 
that the increase in SCA risk for AAOCA patients, although real, is relatively small, particularly for an anomalous right coronary artery. ${ }^{5}$ Thus, surgical intervention must be less hazardous than natural history, while also durably effective. This report offers us another step in that direction.

\section{References}

1. Jegatheeswaran A, Devlin PJ, Williams WG, Brothers JA, Jacobs ML, DeCampli WM, et al. Outcomes after anomalous aortic origin of a coronary artery repair: a Congenital Heart Surgeons' Society study. J Thorac Cardiovasc Surg. 2020;160:757-71.e5.
2. Brothers JA, Frommelt MA, Jaquiss RDB, Myerburg RJ, Fraser CD Jr Tweddell JS. Expert consensus guidelines: anomalous aortic origin of a coronary artery. J Thorac Cardiovasc Surg. 2017;153:1440-57.

3. Buratto E, Konstantinov IE. Current surgical management of anomalous aortic origin of a coronary artery. J Thorac Cardiovasc Surg. September 3, 2020 [Epub ahead of print].

4. Bonilla-Ramirez C, Molossi S, Sachdeva S, Reaves-O'Neal D, Masand P, Mery CM, et al. Outcomes in anomalous aortic origin of a coronary artery after surgical reimplantation. J Thorac Cardiovasc Surg. 2021;162: 1191-9.

5. Mosca RS, Phoon CKL. Anomalous aortic origin of a coronary artery is not always a surgical disease. Semin Thorac Cardiovasc Surg Pediatr Card Surg Ann. 2016; 19:30-6.

\section{Commentary: Transection and reimplantation: Putting all your eggs in one basket?}

\author{
Anusha Jegatheeswaran, MD, PhD, FRCSC
}

In the current issue of the Journal, Bonilla-Ramirez and colleauges ${ }^{1}$ present the retrospective single-center results of 16 patients who underwent transection and reimplantation (TAR) within their Coronary Artery Anomalies Program between 2012 and 2019. TAR was their chosen surgical repair strategy for patients with an intramural anomalous aortic origin of the coronary artery(ies) (AAOCA) who could not undergo isolated unroofing due to presence of a coronary pathway below a commissure and with potential compression by the intercoronary pillar, proximity of the orifice to the commissural pillar, or when unroofing alone would not achieve relocation to the appropriate sinus. This primarily descriptive paper attempts to provide the reader with an understanding of the utility of TAR, in

\footnotetext{
From the Division of Cardiovascular Surgery, Department of Surgery, Hospital for Sick Children, University of Toronto, Toronto, Ontario, Canada.

Disclosures: The author reported no conflicts of interest.

The Journal policy requires editors and reviewers to disclose conflicts of interest and to decline handling or reviewing manuscripts for which they may have a conflict of interest. The editors and reviewers of this article have no conflicts of interest.

Received for publication Jan 4, 2021; revisions received Jan 4, 2021; accepted for publication Jan 5, 2021; available ahead of print Jan 19, 2021.

Address for reprints: Anusha Jegatheeswaran, MD, PhD, FRCSC, Division of Cardiovascular Surgery, Hospital for Sick Children, 555 University Ave, Toronto, Ontario M5G 1X8, Canada (E-mail: anusha.jegatheeswaran@utoronto.ca).

J Thorac Cardiovasc Surg 2021;162:1201-2

$0022-5223 / \$ 36.00$

Copyright (c) 2021 by The American Association for Thoracic Surgery

https://doi.org/10.1016/j.jtcvs.2021.01.014
}

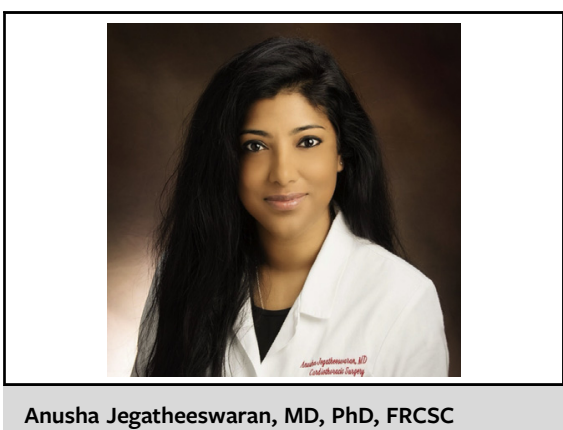

CENTRAL MESSAGE

While transection and reimplantation seem acceptable, it would be a shame to put all our eggs in one basket when we do not have the evidence to suggest any of the available options are superior.

comparison with isolated unroofing, which was used in 45 patients at their institution.

One of the primary strengths of the Coronary Artery Anomalies Program at Texas Children's is that they attempt to evaluate patients in a standardized fashion both pre- and postoperatively. This allows the authors to present standardized data related to outcomes following AAOCA repair, as patients have the same metrics available for evaluation. Important limitations of this manuscript, however, include the very small sample size of 16 patients, which included 6 patients crossing over from an unroofing strategy to a TAR strategy intraoperatively, and the limited follow-up 